\title{
A systematic review for evidence of efficacy of anticholinergic drugs to treat drooling
}

\author{
P H Jongerius, P van Tiel, J van Limbeek, F J M Gabreëls, J J Rotteveel
}

Drooling frequently occurs in children with multiple handicaps; application of anticholinergic drugs is a potential strategy to treat drooling. A computer aided search of original studies concerning the treatment of drooling was carried out. The methodological and statistical integrity of the identified studies were assessed with previously defined criteria. The articles were weighed for their separate contribution to the evidence. The search resulted in 64 reports, of which seven studies passed the screening and were subjected to further assessment and discussion by three referees. Because of the small number of reports and the methodological restriction within the studies, no meta-analysis could be performed. No general conclusion could be made about the efficacy of anticholinergic drugs in treatment of drooling in children with multiple handicaps. There was some evidence that three anticholinergic drugs (benztropine, glycopyrrolate, and benzhexol hydrochloride) are effective in the treatment of drooling, but it could not be concluded that one drug is preferable.

See end of article for authors' affiliations

Correspondence to:
Dr P H Jongerius,
Department of
Rehabilitation, University
Medical Centre
"St Radboud", PO Box
$91016500 H B$, Nijmegen
(internal code 720),
Netherlands;
P.Jongerius@reval.umcn.nl
Accepted
3 January 2003
........................

D rooling has been reported to be a significant problem in about $10-37.5 \%$ of patients with cerebral palsy. ${ }^{1-3}$ Use of anticholinergics is regarded as a possible treatment option. $\mathrm{Nunn}^{4}$ concluded that "the lack of a scientific approach to many of the studies cited makes it virtually impossible to conclude that any one approach is better than another".

The objective of this study was to perform a systematic review of the literature to investigate the efficacy of anticholinergic drugs in the treatment of drooling in children with multiple handicaps.

\section{METHODS}

Search

Articles for review, from 1966 onwards, were identified in Medline, the Cochrane Library, and Current Contents using keywords and the "explode function" present in Medline.

Only patient related studies published in the English, German, Dutch, or French languages were included. Three referees independently analysed all selected studies.

The publications were blinded with respect to author, source, and results. Subsequently the level of methodological quality was assessed. The studies that passed the preliminary screening were subjected to a systematic review using a checklist with previously defined methodological criteria (table 1).

Each criterion was scored with a three level system: [3] sufficient, [2] moderate, [1] insufficient. In case a choice had to be made between sub-items, only one of these could be filled in and the other sub-item scored [0].

\section{RESULTS OF THE LITERATURE SEARCH}

The primary search resulted in 64 articles. Fifty seven, with or without abstracts were rejected. Reasons were: irrelevance to the research question, foreign language, or inadequate methodology. Screening of the references of all articles did not bring up new articles. Seven articles were selected for further investigation (table 2). Three were randomised controlledl trials (RCTs), ${ }^{5-7}$ three were cohort studies, ${ }^{8-10}$ and one was an experimental design. ${ }^{11}$

Two RCTs ${ }^{57}$ and two cohort studies ${ }^{810}$ did not meet the proposed methodological criteria. In order to provide a complete overview of the available literature, all outcomes are listed in table 2.

For methodological quality, the internal validity was regarded as a critical aspect, in particular homogeneity. Randomisation and intention to treat are items that are not applicable for cohort studies. To be qualified as an article with good internal validity, the studies had to satisfy the above mentioned criteria of internal validity with a minimum score of 12 points (out of 21 ) for RCTs or 8 points (out of 15) for cohort studies.

\section{DESCRIPTION OF THE INCLUDED STUDIES}

The articles are described particularly with respect to the methodological quality.

Camp-Bruno et al ${ }^{6}$ investigated the effect of benztropine in a placebo controlled RCT. Homogeneity of the population was rated insufficient because there was no correction for age. Of the 27 patients, seven were later drop outs $(30 \%)$. Unfortunately the outcomes of the measurements were not presented. In spite of these negative points the internal validity scored good: $85.7 \%$ (18/21, meaning 18 out of a maximum of 21 points). External validity: $83.3 \%$; data presentation: $100 \%$. In conclusion, this study could be used for the evidence synthesis.

Abbreviations: NA, not applicable; RCT, randomised controlled trial 
Table 1 Checklist for methodological evaluation of included articles

\begin{tabular}{|c|c|}
\hline \multicolumn{2}{|c|}{ Internal validity (V1-V7) } \\
\hline 1 & Randomisation method presented. \\
\hline $2 a$ & $\begin{array}{l}\text { Homogeneity of the population at entry of the study concerning diagnosis, confounding factors, } \\
\text { prognostic factors. }\end{array}$ \\
\hline $2 b$ & Subgroup analysis done with respect to the mechanism for drooling if necessary. \\
\hline 3 & Description of a method to control for "adherence to therapy". \\
\hline 4 & $\begin{array}{l}\text { Description of a system for control of co-interventions (ENT surgery, behavioural therapy, and } \\
\text { medication) at entry and during the study. }\end{array}$ \\
\hline 5 & Standardised method of outcome measure fully described. \\
\hline 6 & Repeated measurements during the observation period according to a fixed protocol. \\
\hline 7 & Intention to treat analysis if applicable. \\
\hline \multicolumn{2}{|c|}{ External validity (V8-V15) } \\
\hline 8 & Description of inclusion and exclusion criteria. \\
\hline 9 & Accurate description of the planned therapy or interventions. \\
\hline 10 & Check for co-intervention during the trial. \\
\hline 11 & Outcome rates correctly listed in the text. \\
\hline 12 & Description of relevant characteristics related to loss to follow up and adequate management of drop outs. \\
\hline 13 & Presentation of the number of subjects "lost to follow up". \\
\hline 14 & Minimal follow up period of three months. \\
\hline 15 & Control for side effects. \\
\hline \multicolumn{2}{|c|}{ Data presentation (D1-D5) } \\
\hline 1 & Adequate sample size. \\
\hline 2 & Presentation of the mean of the outcome measures. \\
\hline 3 & Presentation of the standard deviation of the outcome measures. \\
\hline 4 & Method of statistical analysis described in relation to the design used. \\
\hline 5 & Appropriate statistical analysis done. \\
\hline
\end{tabular}

The study by Bruno-Camp et al shows that benztropine can have a positive effect on drooling. One cannot make a statement about the average effect nor about adverse effects because of a short follow up period. The population with 27 subjects was too small to compensate for $30 \%$ drop outs. Three of the seven drop outs were certainly related to the treatment.

Mier et $a l^{5}$ evaluated the efficacy and dose ranging effects of glycopyrrolate to treat drooling. The criteria for random- isation, inclusion and exclusion criteria, homogeneity, and "intention to treat" were not satisfied. The number of drop outs $(31 \%)$ is not acceptable because the drop outs appeared to be selectively related to the medication.

Internal validity: $52.3 \%$; external validity: $83.3 \%$; data presentation: $100 \%$. Because of the low score on internal validity this study could only be used in the evidence synthesis to support primary evidence.

Table 2 The methodological assessment of selected studies

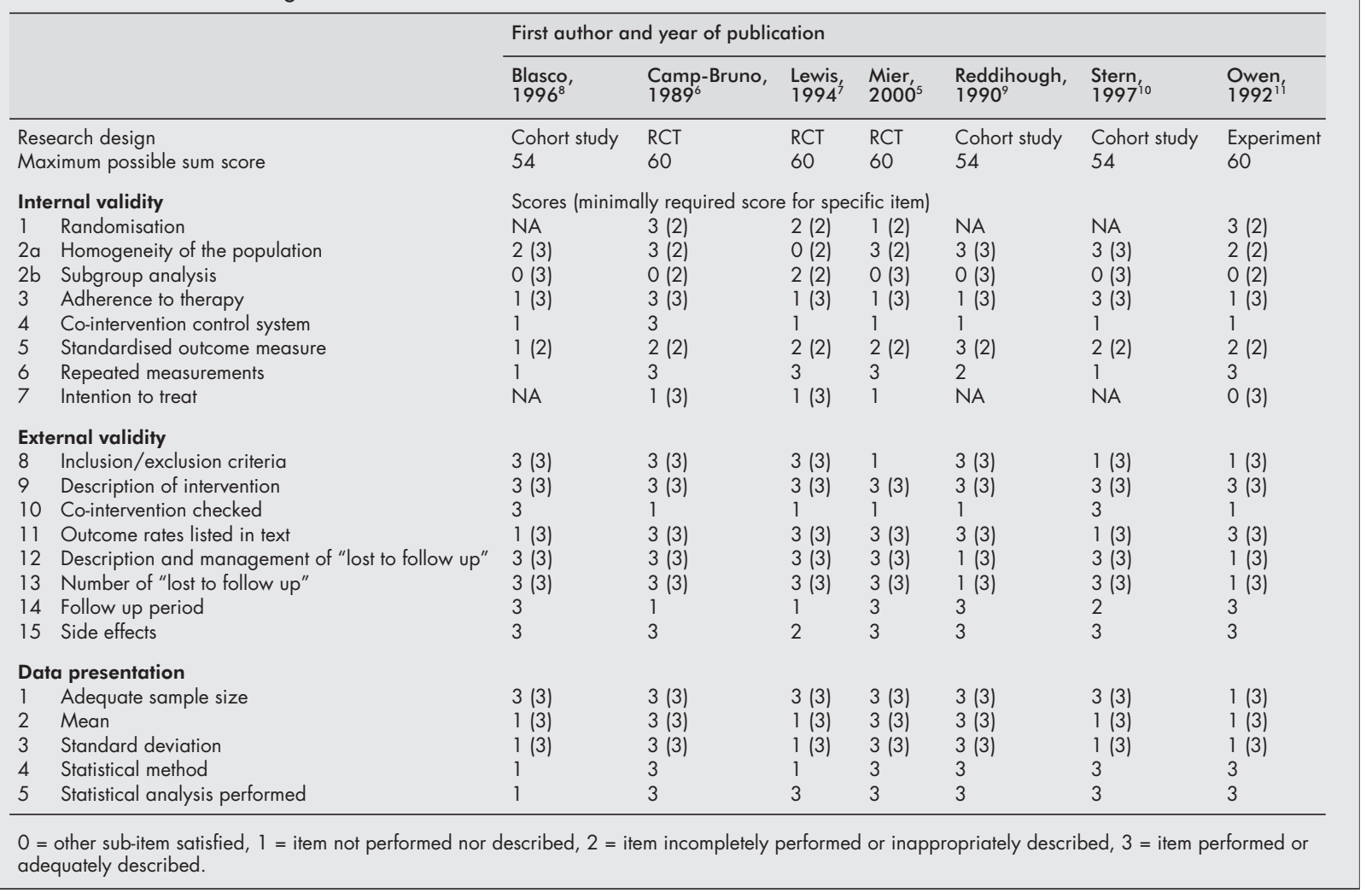


The authors conclude a "marked improvement in drooling". Dosage guidelines are provided. In $20 \%$ of the cases adverse effects necessitated withdrawal of the glycopyrrolate.

Lewis et al ${ }^{7}$ investigated the effect of transdermal application of scopolamine. The homogeneity was insufficient. Adherence to therapy, the "intention to treat", and the method of measurement were insufficiently described. The statistical analysis was not presented in sufficient detail.

Internal validity: $57.1 \%$; external validity: $79.2 \%$; data presentation: $60 \%$. The article could not be used in the evidence synthesis because of the low internal validity in combination with the way data were presented.

The authors presented a good overview of the side effects of scopolamine.

Owen and Stern ${ }^{11}$ investigated the effect of benztropine using a "within subject design". The methodological quality of the study was correct. Because of the small number of patients the study does not permit a judgement as to whether benztropine is a useful therapy, in general. There was insufficient homogeneity in the population. No information was given as to whether statistical analysis was done.

Internal validity: $57.1 \%$; external validity: $66.6 \%$; presentation: $60 \%$. Because of the objective and the chosen research design this study could only be used as additional information to support the evidence.

The study by Owen and Stern indicates that the salivary glands would react to benztropine with a positive effect on salivary flow.

Reddihough et al ${ }^{9}$ studied the effect of benzhexol hydrochloride in a well documented homogeneic population. From the presented results table, it was not clear which data belonged to a particular patient. This might have been of importance with respect to the differences in age.

Internal validity: 66.6\%; external validity: $75 \%$; data presentation: $100 \%$. This cohort study only provides additional information to support the evidence. In the evidence synthesis this study could be used as secondary evidence.

The article gives information about the application of benzhexol hydrochloride and a description of optimal dosage. Because of reasonable methodological quality, two conclusions are likely: (a) benzhexol hydrochloride has a good effect on drooling, although the average effect remains unclear; and (b) the optimal dosage varies from $2 \times 2 \mathrm{mg}$ up to $2 \times 3 \mathrm{mg}$ daily. Blasco and Stansbury investigated the effect of glycopyrrolate. This was a cohort study that did not satisfy the minimal requirements for internal validity. The use of medication was made explicit and half of the population appeared to use a variety of drugs, but no appropriate information was given as to whether these drugs could influence salivary flow. Unfortunately the outcome measures were not listed in the text. In the data presentation baseline measures were not mentioned.

Internal validity: 40\%; external validity: 91.6\%; data presentation: $45.6 \%$. Based on the data set presented in the article, together with the scores on internal validity, no statement could be made about the efficacy of glycopyrrolate.

Although this study cannot be used in the evidence synthesis, the information provided is of clinical importance. The use of anticholinergic drugs and in particular the dosages of glycopyrrolate are presented.

In the study by Stern, ${ }^{10}$ the effect of glycopyrrolate was investigated. It is not possible to determine whether more than $50 \%$ of the population is under the age of 18 . The outcome measures used have limitations; the authors admit that the way questionnaires were completed is open to discussion and criticism. The items on the inclusion and exclusion criteria, homogeneity, and the performed intervention were satisfied. Measurements before the start of the therapy were not listed in the text, nor were the post-treatment results given per patient. Insufficient insight was acquired in the effect of the intervention with glycopyrrolate. Information about statistical analysis provided in the text was inadequate.
Internal validity: 66\%; external validity: $75 \%$; data presentation: $73.3 \%$. As a case series the study could not be taken into account for "evidence synthesis".

From a clinical point of view the authors provide a good overview of the mechanism of drooling and the treatment possibilities, even though this was not the purpose of the article.

\section{DISCUSSION}

RCTs can give primary evidence, whereas cohort studies, referred to as pre-experimental design, can only provide additional information to support the outcome of the RCTs.

One $\mathrm{RCT}^{6}$ acquired sufficient points on internal validity and can be weighed as "high grade evidence". The other RCTs ${ }^{7}$ did not. One $e^{5}$ gives "moderate grade evidence", and one ${ }^{7}$ is a "low grade evidence" study. The experimental study ${ }^{11}$ scored 12 out of 12 possible points on internal validity. Although not an RCT, this study was judged to provide additional information to the primary evidence.

One cohort study" was considered to be a "moderately informative" study. The other two ${ }^{810}$ were regarded as "less informative".

The application of anticholinergic drugs is regarded as a realistic possibility to treat drooling. This systematic review investigated the literature for evidence of the effectiveness of these drugs. An overall problem in the studies is that no single method of measurement of salivary flow and outcome presentation is available. Another problem is that no drug has been repeatedly evaluated. As an outcome of our study no statement can be made about the long term effects because non of the studies describe a follow up period greater than a few weeks. Adverse effects were reported in all studies.

From the selected articles one can conclude that a daily dosage of 3-3.8 mg benztropine could be effective. An impressive reduction in the mean score for drooling was described with benzhexol hydrochloride ( $2 \times 2 \mathrm{mg}$ up to $2 \times 3 \mathrm{mg}$ daily). There is some support of evidence for a marked reduction of drooling with glycopyrrolate.

\section{CONCLUSION}

The objective of this study was to investigate the efficacy of anticholinergic drugs to treat drooling in children with multiple handicaps. We performed an in depth systematic review of the medical literature in order to do a meta-analysis. Unfortunately only seven studies could be identified. Because of the methodological drawbacks within the studies, no general conclusion can be made about the efficacy or average effect of anticholinergic drugs to treat drooling in children with multiple handicaps. Future uniformity in measurements can help the interpretation of outcomes. Based on our study there is some evidence that at least three anticholinergic drugs (benztropine, glycopyrrolate, and benzhexol hydrochloride) are effective in the treatment of drooling, but it cannot be concluded that one anticholinergic drug is preferable to the others. Because of the small number of reports and the methodological restrictions within the studies, no meta-analysis could be performed.

\section{ACKNOWLEDGEMENT}

This work has been supported by a grant from the Johanna KinderFonds: a fund raising association in the field of child rehabilitation, Arnhem, Netherlands.

Authors' affiliations

P H Jongerius, F J M Gabreëls, Department of Rehabilitation University Medical Centre "UMC St Radboud", Nijmegen, Netherlands J J Rotteveel, Department of Pediatric Neurology, University Medical Centre "UMC St Radboud"

P van Tiel, J van Limbeek, Sint Maartenskliniek, Rehabilitation Centre, Nijmegen, Netherlands 
This review is a shortened version of the original review, which can be viewed on the ADC website (www.archdischild.com/supplemental)

\section{REFERENCES}

1 Crysdale WS, White A. Submandibular duct relocation for drooling: a 10-year experience with. Otolaryngol Head Neck Surg 1989:101:87-92.

2 Ekedahl C, Hallen O. Quantitative measurement of drooling. Acta Otolaryngol 1973;75:464-9.

3 Harris SR, Purdy AH. Drooling and its management in cerebral palsy. Dev Med Child Neurol 1987;29:807-1 1.

4 Nunn JH. Drooling: review of the literature and proposals for management. J Oral Rehabil 2000;27:735-43.
5 Mier RJ, Bachrach SJ, Lakin RC, et al. Treatment of sialorrhea with glycopyrrolate: a double-blind, dose-ranging study. Arch Pediatr Adolesc Med 2000;154:1214-18.

6 Camp-Bruno JA, Winsberg BG, Green-Parsons AR, et al. Efficacy of benztropine therapy for drooling. Dev Med Child Neurol

1989:31:309-19.

7 Lewis DW, Fontana C, Mehallick LK, et al. Transdermal scopolamine for reduction of drooling in developmentally delayed children. Dev Med Child Neurol 1994;36:484-6.

8 Blasco PA, Stansbury JC. Glycopyrrolate treatment of chronic drooling Arch Pediatr Adolesc Med 1996;150:932-5.

9 Reddihough D, Johnson H, Staples $M$, et al. Use of benzhexol hydrochloride to control drooling of children with cerebral palsy. Dev Med Child Neurol 1990;32:985-9.

10 Stern LM. Preliminary study of glycopyrrolate in the management of drooling. J Paediatr Child Health 1997;33:52-4.

11 Owen SE, Stern ML. Management of drooling in cerebral palsy: three single case studies. Int J Rehabil Res 1992;15:166-9.

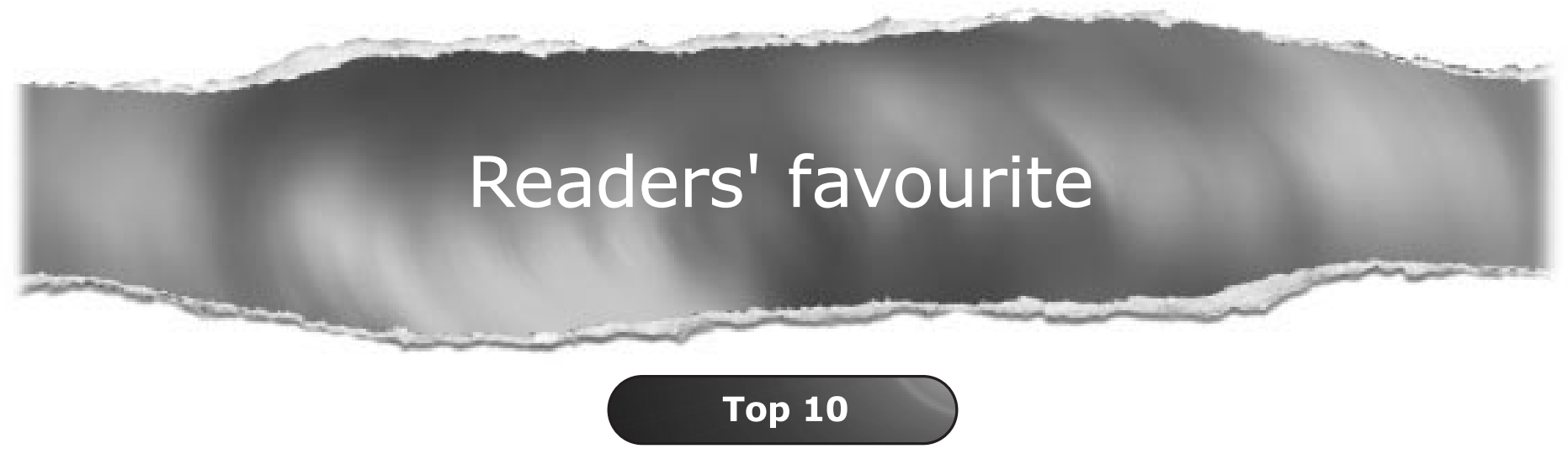

Click on the "Top 10" button on the homepage to see which are the best read articles each month

www.archdischild.com 